\title{
ISOIMMUNE HAEMOLYTIC DISEASE: EFFECT OF IMMUNOGLOBULIN ON HAEMATOLOGICAL PARAMETERS AND SAFETY PROFILE
}

\author{
B. Freyne ${ }^{1}$, F. O'Hare ${ }^{2}$, E. Molloy ${ }^{2,3,4}$ \\ ${ }^{1}$ Neonataology, $\mathrm{NMH}$, Holles St, ${ }^{2}$ Neonatology, $\mathrm{NMH},{ }^{3}$ Medicine \& Medical Sciences, UCD, ${ }^{4}$ Paediatrics, \\ Royal College of Surgeons, Dublin, Ireland
}

IVIG administration to neonates with isoimmune haemolytic disease has been shown to decrease duration of phototherapy and exchange transfusion. IVIG has complex immunodulating effects which are poorly understood. Concerns have been raised that IVIG is associated with necrotising enterocolitis.

To assess the effect of IVIG administration on haematological parameters of neonates with Rhesus disease and to report the safety of this intervention.

74 patients with Rhesus disease were identified over 5 years. FBC parameters were obtained on day 1 and day 3 and pre \& post each dose of IVIG. Any infants with suspected sepsis or a raised CRP was excluded. All analysis was conducted using SPSS 14.0 for windows.

74 infants were treated for Rhesus haemolytic disease, of which 33 received IVIG (58 doses). Mean WCC parameters and $\mathrm{Hb}$ on day $1 \& 3$ were within normal parameters irrespective of treatment given. Statistically rather then clinically significant shifts in individual WCC parameters were noted pre and post individual doses but were not bourne out over the course of treatment. There were no reported adverse effects at time of transfusion. The sole case of NEC did not receive IVIG.

IVIG does not cause clinically significant fluctuations in white cell parameters in neonates. Refractory anaemia requiring readmission was not modified by IVIG administration. IVIG is a safe and effective therapy and there was no evidence of an association with NEC.

\begin{tabular}{|l|l|l|l|l|l||}
\hline & Length of Stay & SBR fraction & Lowest $\mathrm{Hb}$ & $\begin{array}{l}\text { Refractory } \\
\text { Anaemia }\end{array}$ & $\begin{array}{l}\text { Exchange } \\
\text { Transfusion }\end{array}$ \\
\hline No IVIG & $8.3+/-8.9$ & $10.8+/-11.7$ & $14.4+/-21.2$ & 9 & 3 \\
\hline IVIG & $10.2+/-5.3$ & $20.0+/-16.1$ & $11.4+/-14.1$ & 7 & 0 \\
\hline p value & $<.05$ & $<.05$ & $<.05$ & N/A & N/A \\
\hline
\end{tabular}

[Table1: SBR=serum bilirubin. SBR fraction=highest] 